\title{
Acute administration of carnitine to rats modified the basal and A23187-stimulated release of eicosanoids from 4 day carrageenin-elicited peritoneal macrophages
}

\author{
G. R. Elliott ${ }^{1}$, A.P. M. Lauwen ${ }^{2}$ and I. L. Bonta ${ }^{2}$ \\ 1 Pharmacology Department, Medical Biological Laboratory, TNO, PO Box 45, 2280 AA, Rijswijk ZH, The Netherlands \\ (present address) \\ 2 Pharmacology Department, Erasmus University Rotterdam, Postbox 1738, 3000DR, Rotterdam, The Netherlands
}

\begin{abstract}
\section{Introduction}

L-carnitine $\quad(\beta$-hydroxy-(-N-trimethylammonio)butyrate) is a natural amino acid which is essential for the transport of long chain fatty acids into mitochondria for $\beta$-oxidation [1]. Recently carnitine has been shown to enhance the formation of arachidonic acid (AA) from linoleic acid by isolated hepatocytes and to inhibit human neutrophil superoxide production $[2,3]$. As cyclooxygenase (CO) and lipoxygenase ( $\mathrm{LO}$ ) metabolites of AA (eicosanoids) are important modulators of macrophage functions, including superoxide production, [4] we investigated the possibility that L-carnitine could also modulate macrophage eicosanoid synthesis.
\end{abstract}

Carnitine preferentially stimulates the basal and calcium-ionophore (A23187)-stimulated synthesis and

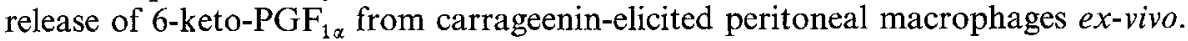

\section{Materials and methods}

Male Wistar rats (16 weeks, four rats/group) were given $300 \mathrm{mg}$ carnitine $/ \mathrm{kg}$ dissolved in $1 \mathrm{ml}$ distilled water, or distilled water alone, by intubation on days 1-4. On day 1 all animals were injected with $2 \mathrm{ml}$ of a carrageenin solution $(1 \mathrm{mg} / \mathrm{ml})$ intraperitoneally. On day $4,1 \mathrm{~h}$ after the last administration of carnitine, the macrophages were isolated from pooled peritoneal washes by density gradient centrifugation and suspended at $2 \times 10^{6}$ nucleated cells $/ \mathrm{ml}$ in Dulbecco's modified Eagle's medium. Portions ( $1 \mathrm{ml}$ ) of the macrophage preparation were incubated at $37^{\circ} \mathrm{C}$ for either $2 \mathrm{~h}$ (basal release) or for $30 \mathrm{~min}$ with $10^{-7} \mathrm{M}$ A23187 (ionophore-stimulated release). The cells were then centrifuged and the supernatant fractions analysed for thromboxane (TX) $\mathrm{A}_{2}$ (analysed as $\mathrm{TXB}_{2}$ ) prostaglandin (PG) $\mathrm{E}_{2}$, prostacyclin $\left(\mathrm{PGI}_{2}\right)$ (analysed as 6 keto-PGF ${ }_{1 \alpha}$ ) and leukotriene (LT) $\mathrm{B}_{4}$ by radioimmunoassay.

In other experiments carrageenin-elicited macrophages from control rats were incubated with carnitine for $2 \mathrm{~h}$ or $24 \mathrm{~h}$. The supernatant fractions were also analysed for eicosanoids.

\section{Results}

Carnitine significantly $(p<0.005)$ reduced the number of macrophages isolated $4 \mathrm{~d}$ after an intraperitoneal injection of carrageenin: control $40 \pm 11$, carnitine $19 \pm 6$ (Values are mean $\pm S D$; $\times 10^{6} ; n=3$ ).

The basal synthesis and release of $\mathrm{PGE}_{2}, 6$ keto$\mathrm{PGF}_{1 \alpha}$ and $\mathrm{LTB}_{4}$ was stimulated by carnitine treatment. In contrast, $\mathrm{TXB}_{2}$ formation was inhibited (Table 1). As a result the 6 keto- $\mathrm{PGF}_{1 \alpha}: \mathrm{TXB}_{2}$ ratio increased (control ratio 0.22; carnitine ratio 0.86). A23187 stimulated the release of the four ei- 
Table 1

The effect of carnitine on the basal and A23187-stimulated re lease of eicosanoids from carrageenin elicited rat peritoneal macrophages. Eicosanoid synthesis and release $\left(\mathrm{ng} / 2 \times 10^{6}\right.$ nucleated cells)

\begin{tabular}{llllll}
\hline Eicosanoid & \multicolumn{2}{l}{ Control } & & \multicolumn{2}{c}{$\mathrm{A} 23187\left(10^{-8} M\right)$} \\
\cline { 2 - 3 } \cline { 5 - 6 } & Control & Carnitine & & Control & Carnitine \\
\hline $\mathrm{TXB}_{2}$ & 5.09 & $2.86^{*}$ & & 7.28 & 8.13 \\
-keto-PGF $_{1 \alpha}$ & 1.19 & $2.26^{*}$ & & 4.95 & $22.26^{*}$ \\
$\mathrm{PGE}_{2}$ & 1.05 & $1.50^{*}$ & & 3.75 & 3.58 \\
$\mathrm{LTB}_{4}$ & 0.065 & $0.104^{*}$ & & 0.838 & $1.644^{*}$ \\
\hline
\end{tabular}

Mean values were significantly different from the corresponding control values (Mann-Whitney U-test): ${ }^{*} p<0.05$.

cosanoids assayed. Synthesis of 6 keto-PGF ${ }_{1 \alpha}$ and $\mathrm{LTB}_{4}$ was further enhanced by carnitine. There was no effect on $\mathrm{PGE}_{2}$ or $\mathrm{TXB}_{2}$ formation. In the presence of $\mathrm{A} 23187$ there was a preferential increase in 6 keto- $\mathrm{PGF}_{1 \alpha}$ synthesis, relative to both $\mathrm{TXB}_{2}$ (control ratio 1.02; carnitine ratio 4.95) and $\mathrm{LTB}_{4}$, (A23187 control ratio 6.70; A23187+ carnitine 15.19) (Table 1).

Carnitine had no effect on macrophage eicosanoid release during incubations of $2 \mathrm{~h}$ or $24 \mathrm{~h}$, (values not shown).

\section{Discussion}

The results presented here clearly show that the acute oral administration of carnitine modified the ability of macrophages to synthesize eicosanoids. It is not clear what mechanisms are involved. No changes in LDH or eicosanoid release were detected after incubating macrophages with carnitine in vitro (data not presented) so that it would appear unlikely that carnitine acted indirectly by damaging the macrophages or directly by stimulating phospholipase, CO or LO activities. It is possible that carnitine stimulated AA formation [2] but that we did not detect this in vitro because we incubated our macrophages in a serum free medium. Of particular interest is the finding that carnitine treatment increased the 6 keto- $\mathrm{PGF}_{1 \alpha}: \mathrm{TXB}_{2}$ ratio, (basal and A23187-stimulated) and 6 keto$\mathrm{PGF}_{1 \alpha}: \mathrm{LTB}_{4}$ ratio (A23187-stimulated). A decrease in the $\mathrm{PGI}_{2}: \mathrm{TXA}_{2}$ ratio is thought to be an important factor in the progression of some cardiovascular diseases [5] while the stimulatory effect of LTs are only maximally observed in the absence of $\mathrm{CO}$ products [6]. Thus, changes in eicosanoid ratios induced by feeding carnitine could have important implications for regulation of inflammatory events in general. This concept is supported by the finding that cell infiltration into the peritoneum, in response to chemotactic stimuli induced by carrageenin, was reduced by carnitine treatment. This fact, together with the report that carnitine inhibits neutrophil superoxide production [3], suggests that carnitine could be anti-inflammatory in vivo.

\section{Acknowledgements}

We wish to thank Sigma-Tau Pharmaceutical Co., Rome, for financing this research.

\section{References}

[1] J. Bremer, Carnitine: Metabolism and functions. Physiological Review 63, 1420-1480 (1983).

[2] B. O. Christopherson and J. Norseth, Arachidonic acid synthesis studied in isolated liver cells. Effects of (-)-carnitine and of (+)-decanoylcarnitine. FEBS Letters 133, 981-204 (1981).

[3] M. L. Schinetti and A. Mazzini, Effects of L-earnitine on human neutrophil activity. International Journal of Tissue Reactions VIII, 199-203 (1986).

[4] R. L. Smith and M. J. Weidemann, Reactive oxygen production associated with arachidonic acid metabolism by peritoneal macrophages. Biochemical and Biophysical Research Communications 97, 973-980 (1980).

[5] R. J. Gryglewski, A. Dembinska-Kiec, A. Zmuda and T. Gryglewska, Prostacyclin and thromboxane $A_{2}$ biosynthesis capacities of heart, arteries and platelets at various stages of experimental atherosclerosis in rabbits. Atherosclerosis 31, 385-394 (1978).

[6] E. J. Schenkelaars and I. L. Bonta, Cyclooxygenase inhibitors promote the leukotriene $C_{4}$ induced release of $\beta$-glucuronidase from rat peritoneal macrophages: prostaglandin $E_{2}$ suppresses. International Journal of Immunopharmacology 8, 305-311 (1986). 